\title{
Homogeneous ignition for a three-step chain-branching reaction model
}

\author{
P. A. Blythe - A. K. Kapila - M. Short
}

Published online: 3 August 2007

(C) Springer Science+Business Media B.V. 2007

$\begin{array}{ll}\text { Erratum to: } & \text { J Eng Math (2006) 56:105-128 } \\ & \text { DOI 10.1007/s10665-006-9055-0 }\end{array}$

Dotted and dash-dotted lines from figures 1-3 in this article were inadvertently erased. The corrected figures appear below.

The online version of the original article can be found under doi: 10.1007/s10665-006-9055-0

\section{P. A. Blythe}

Department of Mechanical Engineering \& Mechanics, Lehigh University,

Bethlehem, PA 18015, USA

A. K. Kapila $(\varangle)$

Rensselaer Polytechnic Institute, Troy, New York 12180, USA

e-mail: kapila@rpi.edu

M. Short

Department of Theoretical and Applied Mechanics, University of Illinois,

Urbana, IL 61801, USA 

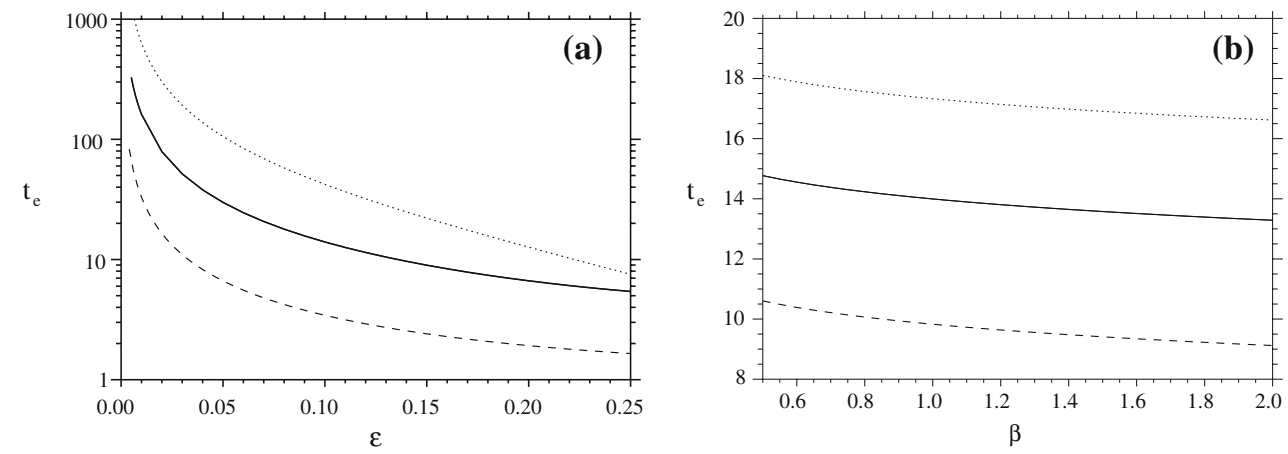

Fig. 1 (a) Variation of time scale for the onset of termination (a) with $\epsilon$ for $m=2.5, T_{i}=3, \beta=1$ and $A=O(1)$, with $A=5$ (dashed line), $A=1$ (solid line) and $A=0.25$ (dotted line); (b) with $\beta$, for $A=1, \epsilon=0.1, m=2.5$ and for $T_{i}=2$ (dashed line), $T_{i}=3$ (solid line), $T_{i}=5$ (dotted line)
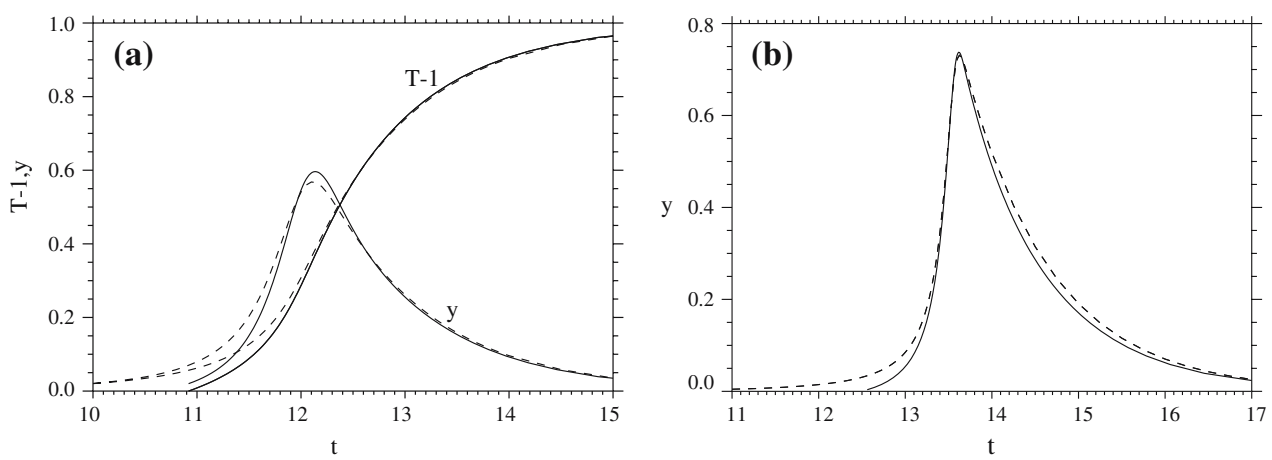

Fig. 2 (a) Comparison with exact (dashed lines) and asymptotic outer composite (solid lines) solutions for $A=O(1)$, for $T_{b}=0.925$, $T_{i}=3, \beta=1, \epsilon=0.125$ and $m=2.5$. (b) Comparison with exact (dashed lines) and asymptotic outer composite (solid lines) solutions for $A=O(1)$ for $y$ for $T_{b}=0.96, T_{i}=3, \beta=1, \epsilon=1 / 16$ and $m=1.5$
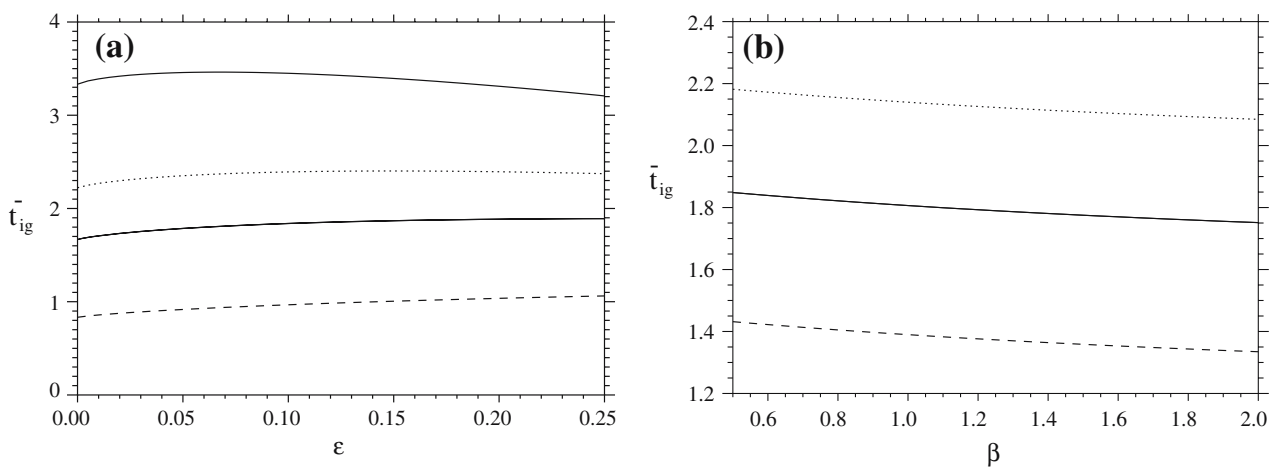

Fig. 3 (a) Ignition time $\bar{t}_{i g}$ as a function of $\epsilon$ for $a=O(1)$ with $m=2.5, T_{i}=3$ and $\beta=0.6$ with $a=2$ (dashed line), $a=1$ (solid line), $a=0.75$ (dotted line) and $a=0.5$ (dot-dash line). (b) Ignition times $\bar{t}_{i g}$ as a function of $\beta$ for $a=1, \epsilon=0.1$ and $m=2.5$ for $T_{i}=2$ (dashed line), $T_{i}=3$ (solid line) and $T_{i}=5$ (dotted line) 